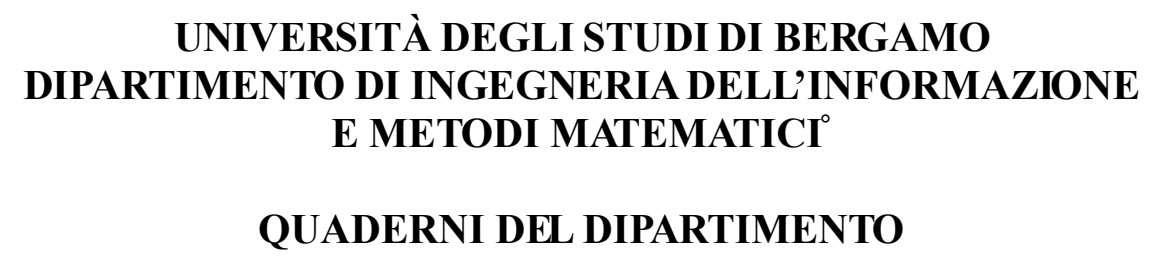

QUADERNI DEL DIPARTIMENTO

Department of Information Technology and Mathematical Methods

Working Paper

Series "Mathematics and Statistics"

n. $12 / \mathrm{MS}-2009$

Nitsche's method for defective boundary value

problems in incompressibile fluid-dynamics

by

Christian Vergara 


\section{COMITATO DI REDAZIONE ${ }^{\S}$}

Series Information Technology (IT): Stefano Paraboschi

Series Mathematics and Statistics (MS): Luca Brandolini, Ilia Negri

\footnotetext{
$\S$ L'accesso alle Series è approvato dal Comitato di Redazione. I Working Papers della Collana dei Quaderni del Dipartimento di Ingegneria dell'Informazione e Metodi Matematici costituiscono un servizio atto a fornire la tempestiva divulgazione dei risultati dell'attività di ricerca, siano essi in forma provvisoria o definitiva.
} 


\title{
Nitsche's method for defective boundary value problems in incompressibile fluid-dynamics
}

\author{
Christian Vergara*
}

\begin{abstract}
In this work we present a unified formulation for the prescription of defective boundary conditions in fluid-dynamics, by means of the Nitsche's method. We study the well-posedness of the discrete problem and the convergence of the numerical solution. Finally, we present several numerical results, focusing on the validation of the proposed method, on a comparison with a pre-existing strategy for the prescription of the flow rate, and on the application to the fluid-structure interaction case.

Keywords: Navier-Stokes equations, defective boundary conditions, Nitsche's method.
\end{abstract}

\section{Introduction}

The prescription of defective boundary conditions in fluid-dynamics has become a problem of great interest in the last decade. In particular, these conditions play a major role when the computational domain is obtained by the truncation of a complex net. Indeed, only average quantities are often available to be prescribed at the artificial sections, that are introduced by the truncation. More precisely, given an artificial section $\Gamma$, three defective conditions have been considered so

${ }^{*}$ Department of Information Technology and Mathematical Methods, Università degli Studi di Bergamo, Viale Marconi 5, 24044 Dalmine (BG), Italy. Email: christian.vergara@unibg.it. Tel.: +39035 2052314. Fax: +39035 562779. This work has been (partially) supported by the ERC Advanced Grant N.227058 MATHCARD and by the Italian MURST, through a project COFIN07. Suggested running head: Nitsche's method for defective problems in fluid-dynamics. 
far, namely

$$
\begin{array}{ll}
\rho_{f} \int_{\Gamma} \boldsymbol{u} \cdot \boldsymbol{n} d \sigma=Q, & \text { Flow rate condition; } \\
\frac{1}{|\Gamma|} \int_{\Gamma} p d \sigma=P, & \text { Mean pressure condition; } \\
\frac{1}{|\Gamma|} \int_{\Gamma}(p-(\mu \nabla \boldsymbol{u} \boldsymbol{n}) \cdot \boldsymbol{n}) d \sigma=P, & \text { Mean normal stress condition }
\end{array}
$$

where $\boldsymbol{u}$ and $p$ are fluid velocity and pressure, $\boldsymbol{n}$ is the outward unit normal on $\Gamma, \rho_{f}$ is the fluid density and $Q$ and $P$ are given data. Navier-Stokes equations equipped with these boundary conditions do not feature well-posedness, since pointwise conditions are needed. Therefore, a suitable mathematical treatment of such conditions is mandatory.

The first ad hoc treatment of defective conditions has been introduced in [15], where a suitable variational formulation has been considered for each defective problem. Afterwards, defective problems have been also treated by the introduction of Lagrange multipliers (see $[4,10,27,28]$ ), or by means of functional minimization (see [13]). In [19] a method based on the weak imposition of a selected velocity profile has been introduced to prescribe a resistance condition (that is a linear combination between the flow rate and the mean pressure) at the outlets. Recently, in [29] it has been proposed to prescribe a flow rate condition by means of a "defective" Nitsche's method [20], with the introduction in the weak formulation of a penalization term. Among these strategies, some of them were introduced for a particular defective problem (such as the methods based on the Lagrange multipliers or on the introduction of a penalization), whilst the methods proposed in [15] and [13] allowed to treat different defective problems. However, in the latter two cases, different defective problems led to different formulations. Therefore, a strategy able to treat different defective problems, with a unified formulation, is still missing.

In the context of pointwise boundary conditions, in [18] the classical Nitsche's method has been extended, for the Possion problem, to the prescription of both Dirichlet and Neumann data at the same time (we refer in the sequel to this approach as extended Nitsche's method). More precisely, the authors have shown that, if the Dirichlet and the Neumann data are compatible, then there exists a unique discrete solution which converges to the solution of the strong problem.

In this paper, we extend the method proposed in [18] to defective problems in fluid-dynamics, obtaining a unified formulation. In particular, we show how it is possible to prescribe, at the same time, the flow rate and the mean normal stress (or the mean pressure) at a certain artificial section, and to switch from a defective problem to another by simply tunining a parameter.

The outline of the paper is as follows. In Section 2 we introduce the new 
formulation and we show that it is consistent. The well-posedness together with the convergence analysis are provided in Section 3. In Section 4 we discuss the case of the prescription of a mean pressure datum. In Section 5 we introduce the Augmented formulation, proposed in $[10,27]$ for the prescription of the flow rate, and we discuss some algebraic issues. Finally, in Section 6 we present several numerical experiments.

\section{Problem setting}

Let $\Omega \subset \mathbb{R}^{d}$ be a truncated computational domain, where $d=2,3$ is the space dimension and the artificial sections are denoted by $\Gamma_{j}$. In Figure 1 we show an example of such a geometry with four artificial sections. For the sake of

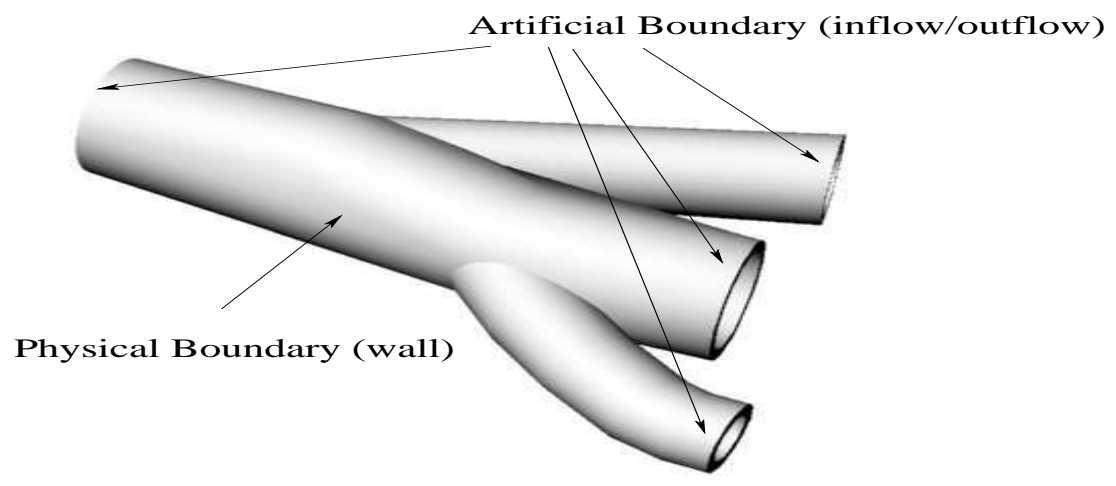

Figure 1: Example of truncated computational domain.

simplicity, in the derivation of the method we focus on a single artificial boundary $\Gamma$ and on homogeneous Dirichlet boundary conditions on the rest of the boundary $\Sigma=\partial \Omega \backslash \Gamma$. The extension of the proposed methodology to an arbitrary number of artificial sections and to non homogeneous/mixed conditions on the rest of the boundary is straightforward. Moreover, in the sequel we set $\rho_{f}=1$.

\subsection{Strong formulation of the defective problem}

We consider the following steady Stokes problem: Given $\varepsilon \geq 0$, find $\boldsymbol{u}$ and $p$ such that

$$
\begin{cases}\mu \triangle \boldsymbol{u}+\nabla p=\boldsymbol{f} & \text { in } \Omega, \\ \nabla \cdot \boldsymbol{u}=0 & \text { in } \Omega, \\ \frac{1}{|\Gamma|} \int_{\Gamma}(-p+\mu \nabla \boldsymbol{u} \boldsymbol{n} \cdot \boldsymbol{n}) d \sigma+\frac{1}{\varepsilon|\Gamma|} \int_{\Gamma} \boldsymbol{u} \cdot \boldsymbol{n} d \sigma=-P+\frac{1}{\varepsilon|\Gamma|} Q, & \\ \boldsymbol{u}=\mathbf{0} & \text { on } \Sigma,\end{cases}
$$


where $\boldsymbol{f}: \Omega \rightarrow \mathbb{R}^{d}$ is a given function and $P$ and $Q$ are given real numbers. Condition $(2)_{3}$ is a defective boundary condition obtained by the linear combination of a mean normal stress and of a flow rate boundary condition. Such a condition can occur in many applications. For example, in haemodynamics (which inspires the present work) the flow rate and the mean normal stress could be known from medical measurements at the same time.

Remark 1. In [15] it has been pointed out that if $\Gamma$ is a plane section perpendicular to a cylindrical pipe, the viscous term in the mean normal stress condition $(1)_{3}$ vanishes identically and the latter reduces to a mean pressure boundary condition $(1)_{2}$. However, for general geometries, there are contexts where the mean normal stress is known, whilst in other situations one knows the mean pressure (for example, in haemodynamics, the first quantity is available from medical measurements, whilst the latter in the geometrical multiscale approach, see, e.g., [9]). For this reason, in this work we treat both these defective conditions (see Section 4).

We introduce the following

Hypothesis 1. The normal stress on $\Gamma$ is aligned with the normal direction and it is constant over the section, that is

$$
-p \boldsymbol{n}+\mu \nabla \boldsymbol{u} \boldsymbol{n}=c \boldsymbol{n}, \quad \text { on } \Gamma,
$$

for a suitable $c \in \mathbb{R}$.

The existence of a unique scalar $c_{P}$ such that the pure mean normal stress problem $\left(\varepsilon \rightarrow \infty\right.$ in $\left.(2)_{3}\right)$ has a unique solution under Hypothesis 1 , has been proved in [15]. For the pure flow rate problem $\left(\varepsilon=0\right.$ in $\left.(2)_{3}\right)$, the existence of a unique scalar $c_{Q}$ has been proved in [26].

Next, we discuss the compatibility of data $P$ and $Q$. To this aim, we introduce the operator $S: \mathbb{R} \rightarrow \mathbb{R}$, as follows: If $Q$ is a given datum, $P=S(Q):=$ $\frac{1}{|\Gamma|} \int_{\Gamma}\left(p_{Q}-\mu \nabla \boldsymbol{u}_{Q} \boldsymbol{n} \cdot \boldsymbol{n}\right) d \sigma$, where $\left(\boldsymbol{u}_{Q}, p_{Q}\right)$ is the unique solution under Hypothesis 1 of system (2) with $\varepsilon=0$ (this is a "defective" Steklov-Poincaré operator). We have the following

Lemma 1. Under Hypothesis 1, the operator $S$ is invertible.

Proof. Let $P \in \mathbb{R}$ be given. Thanks to Hypothesis 1, problem (2) with $\varepsilon \rightarrow \infty$ can be thought as a Neumann problem with datum $c \boldsymbol{n}$ at $\Gamma$. Therefore, it admits a unique solution $\left(\boldsymbol{u}_{P}, p_{P}\right)$ (see, e.g., [14]). By setting $Q:=\int_{\Gamma} \boldsymbol{u}_{P} \cdot \boldsymbol{n} d \sigma$, we have the invertibility of $S$.

The previous Lemma assures that given a datum $Q$, under Hypothesis 1 there exists a unique compatible datum $P$ such that condition $(2)_{3}$ is admissible, and viceversa. From now on, we consider only compatible data. 
Of course, problem (2) has not a unique solution. Among all possible solutions, we focus on the one obtained by making Hypothesis 1. In particular, we have the following

Lemma 2. Under Hypothesis 1, there exists a unique scalar c such that problem (2) admits a unique solution.

Proof. Let $c_{Q}$ and $c_{P}$ be the scalars that assure existence and uniqueness of a solution of the flow rate and of the mean normal stress problems under Hypothesis 1 . Then, for the linear problem (2), existence and uniqueness of a solution easily follow by taking $c:=c_{P}+\frac{1}{\varepsilon|\Gamma|} c_{Q}$ and by superposition of the effects .

\subsection{Weak imposition by means of the extended Nitsche's method}

Here, we extend the formulation proposed in [18] to defective conditions in fluiddynamics. In this context, the flow rate plays the role of defective Dirichlet condition and the mean normal stress of defective Neumann condition.

As observed in [29], thanks to Hypothesis 1, it holds true that

$$
\begin{gathered}
\int_{\Gamma}(-p \boldsymbol{n}+\mu \nabla \boldsymbol{u} \boldsymbol{n}) \cdot \boldsymbol{v} d \sigma=c \int_{\Gamma} \boldsymbol{v} \cdot \boldsymbol{n} d \sigma=\frac{1}{|\Gamma|} \int_{\Gamma}\left(-p+\mu \partial_{n} u_{n}\right) d \sigma \int_{\Gamma} \boldsymbol{v} \cdot \boldsymbol{n} d \sigma= \\
=-\frac{1}{|\Gamma|} \int_{\Gamma} p d \sigma \int_{\Gamma} \boldsymbol{v} \cdot \boldsymbol{n} d \sigma+\frac{1}{|\Gamma|} \int_{\Gamma} \mu \partial_{n} u_{n} d \sigma \int_{\Gamma} \boldsymbol{v} \cdot \boldsymbol{n} d \sigma
\end{gathered}
$$

where, given a velocity field $\boldsymbol{v}$, we have set $\partial_{n} v_{n}:=(\nabla \boldsymbol{v} \boldsymbol{n}) \cdot \boldsymbol{n}$.

Let $\mathcal{T}_{h}$ be a quasi-uniform partition of the domain $\Omega$ in elements $K$ (triangles or tetrahedra), and let $h<1$ be the characteristic mesh size. Then, given $\gamma>0$, 
we introduce the following bilinear forms:

$$
\begin{aligned}
a(\boldsymbol{u}, \boldsymbol{v})= & \mu \int_{\Omega} \nabla \boldsymbol{u}: \nabla \boldsymbol{v} d \boldsymbol{x}+\frac{1}{\varepsilon+\gamma h} \frac{1}{|\Gamma|} \int_{\Gamma} \boldsymbol{u} \cdot \boldsymbol{n} d \sigma \int_{\Gamma} \boldsymbol{v} \cdot \boldsymbol{n} d \sigma+ \\
& -\frac{\gamma h}{\varepsilon+\gamma h} \frac{1}{|\Gamma|}\left[\int_{\Gamma} \mu \partial_{n} u_{n} d \sigma \int_{\Gamma} \boldsymbol{v} \cdot \boldsymbol{n} d \sigma+\int_{\Gamma} \mu \partial_{n} v_{n} d \sigma \int_{\Gamma} \boldsymbol{u} \cdot \boldsymbol{n} d \sigma\right]+ \\
& -\frac{\varepsilon \gamma h}{\varepsilon+\gamma h} \frac{1}{|\Gamma|} \int_{\Gamma} \mu \partial_{n} u_{n} d \sigma \int_{\Gamma} \mu \partial_{n} v_{n} d \sigma \\
b(q, \boldsymbol{v})= & -\int_{\Omega} p \nabla \cdot \boldsymbol{v} d \boldsymbol{x}+\frac{\gamma h}{\varepsilon+\gamma h} \frac{1}{|\Gamma|} \int_{\Gamma} q d \sigma \int_{\Gamma} \boldsymbol{v} \cdot \boldsymbol{n} d \sigma+ \\
& +\frac{1}{\varepsilon+\gamma h} \frac{1}{|\Gamma|} \int_{\Gamma} q d \sigma \int_{\Gamma} \mu \partial_{n} v_{n} d \sigma \\
c(p, q)= & -\frac{\varepsilon \gamma h}{\varepsilon+\gamma h} \frac{1}{|\Gamma|} \int_{\Gamma} p d \sigma \int_{\Gamma} q d \sigma
\end{aligned}
$$

and the following linear functionals:

$$
\begin{aligned}
F(\boldsymbol{v})= & \int_{\Omega} \boldsymbol{f} \cdot \boldsymbol{v} d \boldsymbol{x}+\frac{1}{\varepsilon+\gamma h} \frac{1}{|\Gamma|} Q \int_{\Gamma} \boldsymbol{v} \cdot \boldsymbol{n} d \sigma-\frac{\gamma h}{\varepsilon+\gamma h} \frac{1}{|\Gamma|} Q \int_{\Gamma} \mu \partial_{n} v_{n} d \sigma+ \\
& +\frac{\varepsilon}{\varepsilon+\gamma h} P \int_{\Gamma} \boldsymbol{v} \cdot \boldsymbol{n} d \sigma-\frac{\varepsilon \gamma h}{\varepsilon+\gamma h} P \int_{\Gamma} \mu \partial_{n} v_{n} d \sigma \\
G(q)= & \frac{\gamma h}{\varepsilon+\gamma h} \frac{1}{|\Gamma|} Q \int_{\Gamma} q d \sigma+\frac{\varepsilon \gamma h}{\varepsilon+\gamma h} P \int_{\Gamma} q d \sigma .
\end{aligned}
$$

We set $V_{h}:=\left\{v \in H^{1}(\Omega):\left.v\right|_{\Sigma}=0\right.$ and $\left.\left.v\right|_{K} \in \mathbb{P}^{r}\right\}$, where $\mathbb{P}^{r}$ is the space of polynomials of degree $r$, and $Q_{h}:=\left\{q \in Q:\left.q\right|_{K} \in \mathbb{P}^{s}\right\}$, where $Q:=L^{2}(\Omega)$ if $\varepsilon>0$, whilst $Q:=\left\{q \in L^{2}(\Omega): \int_{\Omega} q d \boldsymbol{x}=0\right\}$ otherwise. From now on, we assume that $\left[V_{h}\right]^{d}$ and $Q_{h}$ are inf-sup compatible (see [5]). Otherwise, suitable stabilization techniques have to be introduced (see, e.g., [6,17]). Then, we consider the following discrete problem:

Problem 1. Given $\boldsymbol{f} \in\left[L^{2}(\Omega)\right]^{d}, Q \in \mathbb{R}$ and $P \in \mathbb{R}$, find $\boldsymbol{u}_{h} \in\left[V_{h}\right]^{d}$ and $p_{h} \in Q_{h}$ such that

$$
\left\{\begin{array}{l}
a\left(\boldsymbol{u}_{h}, \boldsymbol{v}_{h}\right)+b\left(p_{h}, \boldsymbol{v}_{h}\right)=F\left(\boldsymbol{v}_{h}\right) \quad \forall \boldsymbol{v}_{h} \in\left[V_{h}\right]^{d} \\
b\left(q_{h}, \boldsymbol{u}_{h}\right)+c\left(p_{h}, q_{h}\right)=G(q) \quad \forall q_{h} \in Q_{h}
\end{array}\right.
$$

By introducing the following total bilinear form in the couples $(\boldsymbol{u}, p),(\boldsymbol{v}, q)$

$$
A((\boldsymbol{u}, p),(\boldsymbol{v}, q)):=a(\boldsymbol{u}, \boldsymbol{v})+b(p, \boldsymbol{v})+b(q, \boldsymbol{u})+c(p, q),
$$


and the following linear functional in the couple $(\boldsymbol{v}, q)$

$$
L(\boldsymbol{v}, q):=F(\boldsymbol{v})+G(q)
$$

system (6) can be written as

$$
A\left(\left(\boldsymbol{u}_{h}, p_{h}\right),\left(\boldsymbol{v}_{h}, q_{h}\right)\right)=L\left(\boldsymbol{v}_{h}, q_{h}\right) \quad \forall\left(\boldsymbol{v}_{h}, q_{h}\right) \in\left[V_{h}\right]^{d} \times Q_{h} .
$$

We have the following

Theorem 1. Under Hypothesis 1, Problem 1 is consistent with respect to system (2), that is the solution $(\boldsymbol{u}, p)$ of the latter satisfies

$$
A\left((\boldsymbol{u}, p),\left(\boldsymbol{v}_{h}, q_{h}\right)\right)=L\left(\boldsymbol{v}_{h}, q_{h}\right), \quad \forall\left(\boldsymbol{v}_{h}, q_{h}\right) \in\left[V_{h}\right]^{d} \times Q_{h} .
$$

Proof. By multiplying equation $(2)_{1}$ by $\boldsymbol{v}_{h} \in\left[V_{h}\right]^{d}$, integrating over the domain and using the Green's formula, we obtain

$$
\mu \int_{\Omega} \nabla \boldsymbol{u}: \nabla \boldsymbol{v}_{h} d \boldsymbol{x}-\int_{\Omega} p \nabla \cdot \boldsymbol{v}_{h} d \boldsymbol{x}+\int_{\Gamma}(p \boldsymbol{n}-\mu \nabla \boldsymbol{u} \boldsymbol{n}) \cdot \boldsymbol{v}_{h} d \sigma=\int_{\Gamma} \boldsymbol{f} \cdot \boldsymbol{v}_{h} d \sigma
$$

whilst by multiplying equation $(2)_{2}$ by $q_{h} \in Q_{h}$ and integrating over the domain, we obtain

$$
-\int_{\Gamma} q_{h} \nabla \cdot \boldsymbol{u} d \sigma=0
$$

Now, by multiplying the defective condition $(2)_{3}$ by $\frac{\varepsilon}{\varepsilon+\gamma h} \int_{\Gamma} \boldsymbol{v}_{h} \cdot \boldsymbol{n} d \sigma$, we obtain

$$
\begin{gathered}
\frac{\varepsilon}{\varepsilon+\gamma h} \frac{1}{|\Gamma|} \int_{\Gamma}(-p+\mu \nabla \boldsymbol{u} \boldsymbol{n} \cdot \boldsymbol{n}) d \sigma \int_{\Gamma} \boldsymbol{v}_{h} \cdot \boldsymbol{n} d \sigma+ \\
+\frac{1}{\varepsilon+\gamma h} \frac{1}{|\Gamma|} \int_{\Gamma} \boldsymbol{u} \cdot \boldsymbol{n} d \sigma \int_{\Gamma} \boldsymbol{v}_{h} \cdot \boldsymbol{n} d \sigma= \\
=-\frac{\varepsilon}{\varepsilon+\gamma h} P \int_{\Gamma} \boldsymbol{v}_{h} \cdot \boldsymbol{n} d \sigma+\frac{1}{\varepsilon+\gamma h} \frac{1}{|\Gamma|} Q \int_{\Gamma} \boldsymbol{v}_{h} \cdot \boldsymbol{n} d \sigma
\end{gathered}
$$

and similarly, by multiplying it by $\frac{\varepsilon \gamma h}{\varepsilon+\gamma h} \int_{\Gamma}\left(q_{h}-\mu \partial_{n} v_{h, n}\right) d \sigma$, we have

$$
\begin{gathered}
\frac{\varepsilon \gamma h}{\varepsilon+\gamma h} \frac{1}{|\Gamma|} \int_{\Gamma}(-p+\mu \nabla \boldsymbol{u} \boldsymbol{n} \cdot \boldsymbol{n}) d \sigma \int_{\Gamma}\left(q_{h}-\mu \partial_{n} v_{h, n}\right) d \sigma+ \\
\frac{\gamma h}{\varepsilon+\gamma h} \frac{1}{|\Gamma|} \int_{\Gamma} \boldsymbol{u} \cdot \boldsymbol{n} d \sigma \int_{\Gamma}\left(q_{h}-\mu \partial_{n} v_{h, n}\right) d \sigma= \\
=-\frac{\varepsilon \gamma h}{\varepsilon+\gamma h} P \int_{\Gamma}\left(q_{h}-\mu \partial_{n} v_{h, n}\right) d \sigma+\frac{\gamma h}{\varepsilon+\gamma h} \frac{1}{|\Gamma|} Q \int_{\Gamma}\left(q_{h}-\mu \partial_{n} v_{h, n}\right) d \sigma .
\end{gathered}
$$

By summing (8), (9), (10) and (11) together and exploiting Hypothesis 1, we obtain (7) and the thesis follows. 
We rewrite the bilinear form $a(\cdot, \cdot)$ as follows:

$$
\begin{gathered}
a(\boldsymbol{u}, \boldsymbol{v})=\mu \int_{\Omega} \nabla \boldsymbol{u}: \nabla \boldsymbol{v} d \boldsymbol{x}+\frac{1}{\varepsilon+\gamma h} \frac{1}{|\Gamma|} \int_{\Gamma} \boldsymbol{u} \cdot \boldsymbol{n} d \sigma \int_{\Gamma} \boldsymbol{v} \cdot \boldsymbol{n} d \sigma+ \\
-\frac{1}{|\Gamma|}\left[\int_{\Gamma} \mu \partial_{n} u_{n} d \sigma \int_{\Gamma} \boldsymbol{v} \cdot \boldsymbol{n} d \sigma+\int_{\Gamma} \mu \partial_{n} v_{n} d \sigma \int_{\Gamma} \boldsymbol{u} \cdot \boldsymbol{n} d \sigma\right]+ \\
+\frac{\varepsilon}{\varepsilon+\gamma h} \frac{1}{|\Gamma|}\left[\int_{\Gamma} \mu \partial_{n} u_{n} d \sigma \int_{\Gamma} \boldsymbol{v} \cdot \boldsymbol{n} d \sigma+\int_{\Gamma} \mu \partial_{n} v_{n} d \sigma \int_{\Gamma} \boldsymbol{u} \cdot \boldsymbol{n} d \sigma\right]+ \\
-\frac{\varepsilon \gamma h}{\varepsilon+\gamma h} \frac{1}{|\Gamma|} \int_{\Gamma} \mu \partial_{n} u_{n} d \sigma \int_{\Gamma} \mu \partial_{n} v_{n} d \sigma
\end{gathered}
$$

and $b(\cdot, \cdot)$ as follows:

$$
\begin{gathered}
b(q, \boldsymbol{v})=-\int_{\Omega} p \nabla \cdot \boldsymbol{v}_{h} d \boldsymbol{x}+\frac{1}{|\Gamma|} \int_{\Gamma} q d \sigma \int_{\Gamma} \boldsymbol{v} \cdot \boldsymbol{n} d \sigma- \\
\frac{\varepsilon}{\varepsilon+\gamma h} \frac{1}{|\Gamma|} \int_{\Gamma} q d \sigma \int_{\Gamma} \boldsymbol{v} \cdot \boldsymbol{n} d \sigma++\frac{\varepsilon \gamma h}{\varepsilon+\gamma h} \frac{1}{|\Gamma|} \int_{\Gamma} q d \sigma \int_{\Gamma} \mu \partial_{n} v_{n} d \sigma
\end{gathered}
$$

The second term in form $a(\cdot, \cdot)$ together with the second term in functional $F(\cdot)$ can be regarded as the flow rate condition added to the formulation as a penalization. The third term in $a(\cdot, \cdot)$ together with the second term in $b(\cdot, \cdot)$ in the momentum equation are the consistency terms arising from integration by parts of the $L^{2}$-projection of the left hand side of equation (2), that is of $\int_{\Gamma}(-\triangle \boldsymbol{u}+\nabla p) \cdot \boldsymbol{v} d \sigma, \boldsymbol{v} \in\left\{\boldsymbol{w} \in\left[H^{1}(\Omega)\right]^{d}: \boldsymbol{w}=\mathbf{0}\right.$ on $\left.\Sigma\right\}$. The fifth term in $a(\cdot, \cdot)$ and the third term in $b(\cdot, \cdot)$ together with the fourth term in $F(\cdot)$ are added to guarantee the satisfaction of the mean normal stress condition (see Section 2.3 below). The fourth and the sixth term in $a(\cdot, \cdot)$ and the third in $F(\cdot)$ are added in order to obtain a symmetric formulation and do not affect the consistency of the method. The second and the third terms in $b(\cdot, \cdot)$ and the first term in $G(\cdot)$ in the mass equation are added for symmetry purposes and again do not affect the consistency of the method. Term $c(\cdot, \cdot)$ and the last term in $b(\cdot, \cdot)$ together with the second term in $G(\cdot)$ are added to enforce the mean normal stress condition by means of penalization. Finally, to obtain symmetry, the last terms in $a(\cdot, \cdot), b(\cdot, \cdot)$ and in $F(\cdot)$ are added to the momentum equation.

Remark 2. Condition (2) 3 prescribes only the average of the normal velocity and of the normal component of the normal stress. This choice has been inspired by haemodynamics applications, where medical measurements often provide only these averaged data. However, the proposed formulation easily extends to the case of prescription also of the average of the tangential components. In this case, data $Q$ and $P$ are $d$-dimensional vectors. 


\subsection{The case of pure flow rate and mean normal stress conditions}

If we consider the case $\varepsilon=0$ in (6), we have the following discrete problem in the unknowns $\boldsymbol{u}_{h} \in\left[V_{h}\right]^{d}$ and $p_{h} \in Q_{h}$ :

$$
\left\{\begin{aligned}
& \mu \int_{\Omega} \nabla \boldsymbol{u}_{h}: \nabla \boldsymbol{v}_{h} d \boldsymbol{x}+\frac{1}{\gamma h} \frac{1}{|\Gamma|} \int_{\Gamma} \boldsymbol{u} \cdot \boldsymbol{n} d \sigma \int_{\Gamma} \boldsymbol{v} \cdot \boldsymbol{n} d \sigma+ \\
&-\frac{1}{|\Gamma|} \int_{\Gamma} \mu \partial_{n} u_{h, n} d \sigma \int_{\Gamma} \boldsymbol{v}_{h} \cdot \boldsymbol{n} d \sigma-\frac{1}{|\Gamma|} \int_{\Gamma} \mu \partial_{n} v_{h, n} d \sigma \int_{\Gamma} \boldsymbol{u}_{h} \cdot \boldsymbol{n} d \sigma+ \\
&-\int_{\Omega} p_{h} \nabla \cdot \boldsymbol{v}_{h} d \boldsymbol{x}+\frac{1}{|\Gamma|} \int_{\Gamma} p_{h} d \sigma \int_{\Gamma} \boldsymbol{v}_{h} \cdot \boldsymbol{n} d \sigma= \\
&=\int_{\Gamma} \boldsymbol{f} \cdot \boldsymbol{v}_{h} d \sigma+\frac{1}{\gamma h} \frac{1}{|\Gamma|} Q \int_{\Gamma} \boldsymbol{v}_{h} \cdot \boldsymbol{n} d \sigma+\frac{1}{|\Gamma|} Q \int_{\Gamma} \mu \partial_{n} v_{h, n} d \sigma, \forall \boldsymbol{v}_{h} \in\left[V_{h}\right]^{d}, \\
&-\int_{\Omega} q_{h} \nabla \cdot \boldsymbol{u}_{h} d \boldsymbol{x}+\frac{1}{|\Gamma|} \int_{\Gamma} q_{h} d \sigma \int_{\Gamma} \boldsymbol{u}_{h} \cdot \boldsymbol{n} d \sigma=\frac{1}{|\Gamma|} Q \int_{\Gamma} q_{h} d \sigma, \quad \forall q_{h} \in Q_{h},
\end{aligned}\right.
$$

that is nothing but the formulation introduced in [29] for the flow rate problem, where the author proved the stability and convergence of the method.

Conversely, in the case $\varepsilon \rightarrow \infty$ in (6), we obtain the following weak problem

$$
\left\{\begin{aligned}
\mu \int_{\Omega} \nabla \boldsymbol{u}_{h} & : \nabla \boldsymbol{v}_{h} d \boldsymbol{x}-\int_{\Omega} p_{h} \nabla \cdot \boldsymbol{v}_{h} d \boldsymbol{x}-\frac{\gamma h}{|\Gamma|} \int_{\Gamma} \mu \partial_{n} u_{n} d \sigma \int_{\Gamma} \mu \partial_{n} v_{n} d \sigma+ \\
& +\frac{\gamma h}{|\Gamma|} \int_{\Gamma} p d \sigma \int_{\Gamma} \mu \partial_{n} v_{n} d \sigma=\int_{\Gamma} \boldsymbol{f} \cdot \boldsymbol{v}_{h} d \sigma+ \\
& -P \int_{\Gamma} \boldsymbol{v}_{h} \cdot \boldsymbol{n} d \sigma+\gamma h P \int_{\Gamma} \mu \partial_{n} v_{n} d \sigma, \quad \forall \boldsymbol{v}_{h} \in\left[V_{h}\right]^{d}, \\
-\int_{\Omega} q_{h} \nabla & \cdot \boldsymbol{u}_{h} d \boldsymbol{x}-\gamma h \frac{1}{|\Gamma|} \int_{\Gamma} p d \sigma \int_{\Gamma} q d \sigma+\gamma h \frac{1}{|\Gamma|} \int_{\Gamma} \mu \partial_{n} u_{n} d \sigma \int_{\Gamma} q d \sigma= \\
& =-\gamma h P \int_{\Gamma} q d \sigma, \quad \forall q_{h} \in Q_{h} .
\end{aligned}\right.
$$

We observe that in the momentum equation, the third and the fourth terms at the left hand side and the last term at the right hand side impose the mean stress condition $(1)_{3}$ by means of penalization. The same is obtained in the mass conservation equation by the second and third terms at the left hand side and by the right hand side. We remark that the penalization terms appear in both the equations in order to guarantee the symmetry of the method. Moreover, from the first equation of (13), integrating by parts, we obtain again condition $(1)_{3}$, and therefore system (13) is consistent with the mean normal stress condition.

The proposed formulation (6) is then able to treat both the flow rate and the mean normal stress condition, and a linear combination of these. To switch from 
a defective problem to another, it is sufficient to suitably tune the parameter $\varepsilon$.

For the treatment of the mean pressure condition, we refer the reader to Section 4.

\section{Existence of a unique solution and convergence}

To show the existence and uniqueness of a solution of system (6), we start recalling the following theorem.

Theorem 2. Given an abstract system in the form $(6)$, with $F(\cdot)$ and $G(\cdot)$ linear bounded functionals, there exists a unique solution $\left(\boldsymbol{u}_{h}, p_{h}\right) \in\left[V_{h}\right]^{d} \times Q_{h}$ if

a) The bilinear forms $a(\cdot, \cdot), b(\cdot, \cdot)$ and $c(\cdot, \cdot)$ are continuous, that is

$$
\begin{aligned}
& a(\boldsymbol{u}, \boldsymbol{v}) \leq M\|\boldsymbol{u}\|_{\left[V_{h}\right]^{d}}\|\boldsymbol{v}\|_{\left[V_{h}\right]^{d}}, \quad \forall \boldsymbol{u}, \boldsymbol{v} \in\left[V_{h}\right]^{d}, \\
& b(q, \boldsymbol{v}) \leq M\|q\|_{Q_{h}}\|\boldsymbol{v}\|_{\left[V_{h}\right]^{d}}, \quad \forall q \in Q_{h} \text { and } \forall \boldsymbol{v} \in\left[V_{h}\right]^{d}, \\
& c(p, q) \leq M\|p\|_{Q_{h}}\|q\|_{Q_{h}}, \quad \forall p, q \in Q_{h},
\end{aligned}
$$

for suitable constants $M>0$ and for given suitable norms in $\left[V_{h}\right]^{d}$ and $Q_{h}$;

b) The bilinear form $a(\cdot, \cdot)$ is coercive, that is there exists $\alpha>0$ such that

$$
a(\boldsymbol{v}, \boldsymbol{v}) \geq \alpha\|\boldsymbol{v}\|_{\left[V_{h}\right]^{d}}^{2}, \quad \forall \boldsymbol{v} \in\left[V_{h}\right]^{d},
$$

so that its algebraic counterpart is positive definite;

c) The bilinear form $b(\cdot, \cdot)$ satisfies an inf-sup condition: There exists $\beta>0$, such that $\forall q \in Q_{h}$, there exists $v \in\left[V_{h}\right]^{d}$ such that

$$
b(q, \boldsymbol{v}) \geq \beta\|q\|_{Q_{h}}\|\boldsymbol{v}\|_{\left[V_{h}\right]^{d}} .
$$

This means that the algebraic counterpart of $b(\cdot, \cdot)$ has full rank;

d) Form $c(\cdot, \cdot)$ is symmetric and satisfies

$$
c(p, q) \leq 0, \quad \forall p, q \in Q_{h}
$$

that is its algebraic counterpart is symmetric negative semi-definite.

For a proof of the previous theorem we refer the reader, e.g., to [3].

From now on, $C$ denotes a positive constant independent of $\varepsilon$ and $h$. We introduce the following semi-norm

$$
|v|_{\Gamma}^{2}:=\frac{1}{|\Gamma|}\left(\int_{\Gamma} v d \sigma\right)^{2}, \quad v \in H^{1}(\Omega),
$$


and the following norms

$$
\begin{aligned}
\|w\|^{2} & :=\int_{\Omega} w^{2} d \boldsymbol{x}, \quad w \in L^{2}(\Omega) \\
\|\boldsymbol{v}\|_{h}^{2} & :=\|\nabla \boldsymbol{v}\|^{2}+\frac{1}{\varepsilon+\gamma h}|\boldsymbol{v} \cdot \boldsymbol{n}|_{\Gamma}^{2}, \quad \boldsymbol{v} \in\left[H^{1}(\Omega)\right]^{d} .
\end{aligned}
$$

Moreover, recalling the following inequalities, holding for $v \in H^{1}(\Omega)$ (see, e.g., $[25])$,

$$
\int_{\Gamma} v^{2} d \sigma \leq C h^{-1}\|v\|^{2}, \quad|v|_{\Gamma}^{2} \leq \int_{\Gamma} v^{2} d \sigma
$$

we obtain

$$
|v|_{\Gamma} \leq C h^{-1 / 2}\|v\|, \quad \forall v \in H^{1}(\Omega) .
$$

Next, we show that bilinear forms defined in (4) satisfy the assumptions of Theorem 2.

Proposition 1. The bilinear forms $a(\cdot, \cdot), b(\cdot, \cdot)$ and $c(\cdot, \cdot)$ defined in (4) are continuous with respect to norms (14), with constants independent of $h$ and $\varepsilon$.

Proof. Thanks to the Schwarz inequality and (15), we have

$$
\begin{gathered}
a(\boldsymbol{u}, \boldsymbol{v}) \leq \mu\|\nabla \boldsymbol{u}\|\|\nabla \boldsymbol{v}\|+\frac{1}{\varepsilon+\gamma h}|\boldsymbol{u} \cdot \boldsymbol{n}|_{\Gamma}|\boldsymbol{v} \cdot \boldsymbol{n}|_{\Gamma}+ \\
+\frac{\gamma \mu h}{\varepsilon+\gamma h}\left(\left|\partial_{n} u_{n}\right|_{\Gamma}|\boldsymbol{v} \cdot \boldsymbol{n}|_{\Gamma}+\left|\partial_{n} v_{n}\right|_{\Gamma}|\boldsymbol{u} \cdot \boldsymbol{n}|_{\Gamma}\right)+\frac{\varepsilon \gamma \mu^{2} h}{\varepsilon+\gamma h}\left|\partial_{n} u_{n}\right|_{\Gamma}\left|\partial_{n} v_{n}\right|_{\Gamma} \leq \\
\leq \mu\|\nabla \boldsymbol{u}\|\|\nabla \boldsymbol{v}\| \frac{1}{\varepsilon+\gamma h}|\boldsymbol{u} \cdot \boldsymbol{n}|_{\Gamma}|\boldsymbol{v} \cdot \boldsymbol{n}|_{\Gamma}+ \\
+\frac{\gamma \mu h}{\varepsilon+\gamma h} C h^{-1 / 2}\left(\|\nabla \boldsymbol{u}\||\boldsymbol{v} \cdot \boldsymbol{n}|_{\Gamma}+\|\nabla \boldsymbol{v}\||\boldsymbol{u} \cdot \boldsymbol{n}|_{\Gamma}\right)+ \\
+\frac{C \varepsilon \gamma \mu^{2}}{\varepsilon+\gamma h}\|\nabla \boldsymbol{u}\|\|\nabla \boldsymbol{v}\| \leq \\
\leq \mu\|\boldsymbol{u}\|_{h}\|\boldsymbol{v}\|_{h}+\|\boldsymbol{u}\|_{h}\|\boldsymbol{v}\|_{h}+2 C \gamma \mu h^{1 / 2}\|\boldsymbol{u}\|_{h}\|\boldsymbol{v}\|_{h}+C \gamma \mu^{2}\|\boldsymbol{u}\|_{h}\|\boldsymbol{v}\|_{h} \leq \\
\leq\left(\mu+1+2 C \gamma \mu+C \gamma \mu^{2}\right)\|\boldsymbol{u}\|_{h}\|\boldsymbol{v}\|_{h},
\end{gathered}
$$

where in the last inequality we have exploited the fact that $h^{1 / 2}<1$.

Moreover, thanks to the Schwarz and the Poincarè inequalities and (15), we obtain

$$
\begin{gathered}
b(q, \boldsymbol{v}) \leq\|q\|\|\nabla \boldsymbol{v}\|+\frac{\gamma h}{\varepsilon+\gamma h}|q|_{\Gamma}|\boldsymbol{v} \cdot \boldsymbol{n}|_{\Gamma}+\frac{\varepsilon \gamma h}{\varepsilon+\gamma h}|q|_{\Gamma}\left|\mu \partial_{n} v_{n}\right|_{\Gamma} \leq \\
\leq\|q\|\|\nabla \boldsymbol{v}\|+\frac{\gamma h}{\varepsilon+\gamma h} C h^{-1 / 2}\|q\||\boldsymbol{v} \cdot \boldsymbol{n}|_{\Gamma}+\frac{\varepsilon \gamma h}{\varepsilon+\gamma h} C \mu h^{-1}\|q\|\|\nabla \boldsymbol{v}\| \leq
\end{gathered}
$$




$$
\begin{aligned}
\leq\|q\|\|\boldsymbol{v}\|_{h}+ & C \gamma h^{1 / 2}\|q\|\|\boldsymbol{v}\|_{h}+\frac{\varepsilon \gamma}{\varepsilon+\gamma h} C \mu\|q\|\|\nabla \boldsymbol{v}\| \leq \\
& \leq(1+C \gamma+C \gamma \mu)\|q\|\|\boldsymbol{v}\|_{h},
\end{aligned}
$$

where in the last inequality we have observed that $\frac{\varepsilon}{\varepsilon+\gamma h} \leq 1$.

Finally, from the Schwartz inequality and (15), we have

$$
c(p, q) \leq \frac{\varepsilon \gamma h}{\varepsilon+\gamma h}|p|_{\Gamma}|q|_{\Gamma} \leq \frac{\varepsilon \gamma}{\varepsilon+\gamma h} C\|p\|\|q\| \leq C \gamma\|p\|\|q\| .
$$

Proposition 2. There exists a positive constant $C$ independent of $h$ and $\varepsilon$ such that, if

$$
0<\gamma<\frac{1}{\mu C}
$$

then there exists a positive constant $\alpha$ independent of $h$ and $\varepsilon$ such that

$$
a(\boldsymbol{v}, \boldsymbol{v}) \geq \alpha\|\boldsymbol{v}\|_{h}^{2},
$$

for all $\boldsymbol{v} \in\left[V_{h}\right]^{d}$.

Proof. We have, for all $\boldsymbol{v} \in\left[V_{h}\right]^{d}$,

$$
\begin{gathered}
a(\boldsymbol{v}, \boldsymbol{v})=\mu\|\nabla \boldsymbol{v}\|^{2}+\frac{1}{\varepsilon+\gamma h}|\boldsymbol{v} \cdot \boldsymbol{n}|_{\Gamma}^{2}+ \\
-\frac{2 \gamma h \mu}{|\Gamma|(\varepsilon+\gamma h)} \int_{\Gamma} \partial_{n} v_{n} d \sigma \int_{\Gamma} \boldsymbol{v} \cdot \boldsymbol{n} d \sigma-\frac{\varepsilon \gamma h}{\varepsilon+\gamma h} \mu^{2}\left|\partial_{n} v_{n}\right|_{\Gamma}^{2} .
\end{gathered}
$$

Following [18], applying the Schwarz and Young inequalities (with constant $\delta / 2$ ) to the third term above, we obtain

$$
\begin{gathered}
a(\boldsymbol{v}, \boldsymbol{v}) \geq \mu\|\nabla \boldsymbol{v}\|^{2}+\frac{1}{\varepsilon+\gamma h}|\boldsymbol{v} \cdot \boldsymbol{n}|_{\Gamma}^{2}-\frac{2 \gamma h \mu}{\varepsilon+\gamma h}\left|\partial_{n} v_{n}\right|_{\Gamma}|\boldsymbol{v} \cdot \boldsymbol{n}|_{\Gamma}-\frac{\varepsilon \gamma \mu^{2} h}{\varepsilon+\gamma h}\left|\partial_{n} v_{n}\right|_{\Gamma}^{2} \geq \\
\geq \mu\|\nabla \boldsymbol{v}\|^{2}+\frac{1}{\varepsilon+\gamma h}|\boldsymbol{v} \cdot \boldsymbol{n}|_{\Gamma}^{2}-\left(\frac{\mu^{2} \gamma^{2} h^{2}}{\delta(\varepsilon+\gamma h)}+\frac{\varepsilon \gamma \mu^{2} h}{\varepsilon+\gamma h}\right)\left|\partial_{n} v_{n}\right|_{\Gamma}^{2}-\frac{\delta}{\varepsilon+\gamma h}|\boldsymbol{v} \cdot \boldsymbol{n}|_{\Gamma}^{2} \geq \\
\quad \geq \mu\left(1-\frac{\mu \gamma^{2} C h}{\delta(\varepsilon+\gamma h)}-\frac{\varepsilon \gamma \mu C}{\varepsilon+\gamma h}\right)\|\nabla \boldsymbol{v}\|^{2}+\frac{1-\delta}{\varepsilon+\gamma h}|\boldsymbol{v} \cdot \boldsymbol{n}|_{\Gamma}^{2}= \\
=\frac{\mu}{\varepsilon+\gamma h}\left(\varepsilon(1-C \gamma \mu)+\gamma h\left(1-\frac{C \gamma \mu}{\delta}\right)\right)\|\nabla \boldsymbol{v}\|^{2}+\frac{1-\delta}{\varepsilon+\gamma h}|\boldsymbol{v} \cdot \boldsymbol{n}|_{\Gamma}^{2} .
\end{gathered}
$$

The first term in the last expression is positive for $\delta>\mu \gamma C$ and $\gamma<\frac{1}{\mu C}$. The second term is positive for $\delta<1$, so that, thanks to assumption (16), it is possible to choose $\delta$ such that $\mu \gamma C<\delta<1$. Therefore, the thesis follows with

$$
\alpha=\min \left\{\mu\left(1-\frac{C \gamma \mu}{\delta}\right) ; 1-\delta\right\}>0,
$$

which is independent of $\varepsilon$ and $h$. 
For what concerns point $c$ ) in Theorem 2, we introduce the following assumption:

Hypothesis 2. We suppose that an inf-sup condition holds for the classical Stokes problem, that is there exists $\beta>0$ such that for all $q \in Q_{h}$ there exists $\boldsymbol{v} \in\left[V_{h}\right]^{d}$ such that $-\int_{\Gamma} q \nabla \cdot \boldsymbol{v} d \sigma \geq \beta\|q\|\|\nabla \boldsymbol{v}\|$ (see, e.g., [5]). Then, $\boldsymbol{v} \in$ $\left[H_{0}^{1}(\Omega)\right]^{d}$.

This assumption holds true for example for the Taylor-Hood and the MINI elements (see [5]).

We have the following

Proposition 3. If Hypothesis 2 holds true, there exists a positive constant $C$ independent of $h$ and $\varepsilon$ such that, if

$$
0<\gamma<\frac{\beta}{\mu C},
$$

then the bilinear form $b(\cdot, \cdot)$ defined in $(4)_{2}$ satisfies an inf-sup condition with constant independent of $h$ and $\varepsilon$.

Proof. Given $q \in Q_{h}$, we choose $\boldsymbol{v} \in\left[V_{h}\right]^{d} \cap\left[H_{0}^{1}(\Omega)\right]^{d}$ such that Hypothesis 2 holds. We have

$$
\begin{gathered}
b(q, \boldsymbol{v}) \geq \beta\|q\|\|\nabla \boldsymbol{v}\|+\frac{\gamma h}{\varepsilon+\gamma h} \frac{1}{|\Gamma|} \int_{\Gamma} q d \sigma \int_{\Gamma} \boldsymbol{v} \cdot \boldsymbol{n} d \sigma+ \\
+\frac{\varepsilon \gamma h}{\varepsilon+\gamma h} \frac{1}{|\Gamma|} \int_{\Gamma} q d \sigma \int_{\Gamma} \mu \partial_{n} v_{n} d \sigma
\end{gathered}
$$

First of all we highlight that, as observed in [29], since Hypothesis 2 holds, the second term of the right hand side in the previous inequality vanishes. Moreover, thanks to the Schwarz inequality and (15), we obtain

$$
\begin{aligned}
b(q, \boldsymbol{v}) \geq & \beta\|q\|\|\nabla \boldsymbol{v}\|-\left|\frac{\varepsilon \gamma h}{\varepsilon+\gamma h} \frac{1}{|\Gamma|} \int_{\Gamma} q d \sigma \int_{\Gamma} \mu \partial_{n} v_{n} d \sigma\right| \geq \\
& \geq \beta\|q\|\|\nabla \boldsymbol{v}\|-\frac{\varepsilon \gamma h}{\varepsilon+\gamma h}|q|_{\Gamma}\left|\mu \partial_{n} v_{n}\right|_{\Gamma} \geq \\
\geq & \left(\beta-\frac{\varepsilon \gamma \mu C}{\varepsilon+\gamma h}\right)\|q\|\|\nabla \boldsymbol{v}\| \geq \beta^{*}\|q\|\|\nabla \boldsymbol{v}\|,
\end{aligned}
$$

with $\beta^{*}:=\beta-\gamma \mu C$ and where in the last inequality we have observed that $-\frac{\varepsilon}{\varepsilon+\gamma h} \geq-1$. Therefore, for $\gamma<\frac{\beta}{\mu C}$, we have that $\beta^{*}>0 \forall \varepsilon$, and the thesis follows with a constant independent of $h$ and $\varepsilon$, by noticing that, for the chosen function $\boldsymbol{v}$, we have $\|\nabla \boldsymbol{v}\| \equiv\|\boldsymbol{v}\|_{h}$. 
Moreover, we have

$$
c(q, q) \equiv-\frac{\varepsilon \gamma h}{\varepsilon+\gamma h}\|q\|_{\Gamma}^{2} \leq 0 \quad \forall q_{h} \in Q_{h} .
$$

Finally, we have the following

Theorem 3. If Hypothesis 1 and 2 are satisfied, there exists a constant $C$ independent of $h$ and $\varepsilon$ such that, if

$$
0<\gamma<\frac{1}{\mu C} \min \{1 ; \beta\},
$$

then system (6) admits a unique solution $\left(\boldsymbol{u}_{h}, q_{h}\right) \in\left[V_{h}\right]^{d} \times Q_{h}$.

Proof. Thanks to Propositions 1, 2 and 3, inequality (17) and the boundedness of linear functionals (5), the thesis follows by applying Theorem 2 .

In the next theorem we prove that under Hypothesis 1 and 2 the solution of problem (6) converges to the solution of problem (1).

Theorem 4. If the solution of problem (1) satisfies $\boldsymbol{u} \in\left[H^{r+1}(\Omega)\right]^{d}$ and $p \in$ $H^{s+1}(\Omega)$, with $r, s>0$, and if Hypothesis 1 and 2 are satisfied, then there exist two constants independent of $h$ and $\varepsilon$, such that

$$
\left\|\boldsymbol{u}-\boldsymbol{u}_{h}\right\|_{h} \leq C h^{r}|\boldsymbol{u}|_{r+1}, \quad\left\|p-p_{h}\right\| \leq C h^{s+1}|p|_{s+1},
$$

where $\left(\boldsymbol{u}_{h}, p_{h}\right)$ is the solution of problem (6) and $|v|_{s}:=\left\|D^{s} v\right\|$, with $D^{s} v$ the $s-$ th derivative of $v$.

Proof. From the definition of norm $\|\cdot\|_{h}$ and inequality (15), we have

$\|\boldsymbol{v}\|_{h}^{2}=\|\nabla \boldsymbol{v}\|^{2}+\frac{1}{\varepsilon+\gamma h}|\boldsymbol{v} \cdot \boldsymbol{n}|_{\Gamma}^{2} \leq\|\nabla \boldsymbol{v}\|^{2}+\frac{C}{h(\varepsilon+\gamma h)}\|\boldsymbol{v}\|^{2} \leq\|\nabla \boldsymbol{v}\|^{2}+\leq \frac{1}{\gamma h^{2}}\|\boldsymbol{v}\|^{2}$,

where in the last inequality we have taken $\varepsilon=0$. By exploiting the coercivity of $a(\cdot, \cdot)$, the continuity of the forms (4) and the consistency of the problem and by applying the standard interpolation estimates, we obtain the thesis with constants independent of $h$ and $\varepsilon$.

\subsection{The generalized Oseen problem}

Given $\boldsymbol{b} \in\left[L^{\infty}(\Omega)\right]^{d}$, we consider the following momentum equation instead of $(2)_{1}$

$$
\theta \boldsymbol{u}-\mu \triangle \boldsymbol{u}+(\boldsymbol{b} \cdot \nabla) \boldsymbol{u}+\nabla p=\boldsymbol{f} \quad \text { in } \Omega .
$$

This is the case, for example, of the discretized-in-time Navier-Stokes problem if a semi-implicit scheme is used to treat the convective term (see, e.g., [24]). It can 
be easily proved that system (18)-(2) $)_{1,3,4}$ still admits a unique solution under Hypothesis 1 (the existence of scalars $c$ in Hypothesis 1 for the mean normal stress and for the flow rate problems have been again proved in [15] and [26]). Then, we can consider again formulation $(6)$ where $a(\cdot, \cdot)$ is replaced by

$$
a^{G O}(\boldsymbol{u}, \boldsymbol{v})=\theta \int_{\Gamma} \boldsymbol{u} \cdot \boldsymbol{v} d \sigma+a(\boldsymbol{u}, \boldsymbol{v})+\int_{\Gamma}(\boldsymbol{b} \cdot \nabla) \boldsymbol{u} \cdot \boldsymbol{v} d \sigma
$$

We have the following

Corollary 1. If Hypothesis 1 and 2 hold true and

$$
\begin{aligned}
& \mu-C\|\boldsymbol{b}\|_{\infty} \geq \mu^{*}>0 \\
& 0<\gamma<\min \left\{\frac{\mu^{*}}{\mu^{2} C} ; \frac{\beta}{\mu C}\right\},
\end{aligned}
$$

for suitable constants $C>0$ independent of $h$ and $\varepsilon$, then problem (6), with form $a^{G O}(\cdot, \cdot)$, admits a unique solution.

Proof. We follow the same guidelines of the proof of Theorem 3. In particular, we have to show continuity and coercivity of $a^{G O}(\cdot, \cdot)$. Continuity is guaranteed by the fact that $\boldsymbol{b} \in\left[L^{\infty}(\Omega)\right]^{d}$. For coercivity, we have

$$
a^{G O}(\boldsymbol{u}, \boldsymbol{v}) \geq \theta\|\boldsymbol{v}\|^{2}+\alpha\|\boldsymbol{v}\|_{h}^{2}+\int_{\Omega}(\boldsymbol{b} \cdot \nabla) \boldsymbol{v} \cdot \boldsymbol{v} d \boldsymbol{x}
$$

where $\alpha$ is the coercivity constant of $a(\cdot, \cdot)$. For the last term, we obtain

$$
\int_{\Omega}(\boldsymbol{b} \cdot \boldsymbol{v}) \boldsymbol{v} d \boldsymbol{x} \geq-\left|\int_{\Omega}(\boldsymbol{b} \cdot \boldsymbol{v}) \boldsymbol{v} d \boldsymbol{x}\right| \geq-\|\boldsymbol{b}\|_{\infty}\|\nabla \boldsymbol{v}\|\|\boldsymbol{v}\|,
$$

and the coercivity follows thanks to the Poincaré inequality and from hypothesis (19).

\section{Prescribing a mean pressure condition}

In some applications, the mean pressure $(1)_{2}$ is known instead of the mean normal stress $(1)_{3}$, that is we could consider condition

$$
\frac{1}{|\Gamma|} \int_{\Gamma}-p d \sigma+\frac{1}{\varepsilon|\Gamma|} \int_{\Gamma} \boldsymbol{u} \cdot \boldsymbol{n} d \sigma=-P+\frac{1}{\varepsilon|\Gamma|} Q
$$

instead of $(2)_{3}$. This is the case, for example, of haemodynamic applications (see $[10,13,15])$. In such a case, we modify the bilinear form $a(\cdot, \cdot)$ and the 
functional $F(\cdot)$ as follows

$$
\begin{aligned}
a^{P}(\boldsymbol{u}, \boldsymbol{v}):= & \mu \int_{\Omega} \nabla \boldsymbol{u}: \nabla \boldsymbol{v} d \boldsymbol{x}+\frac{1}{\varepsilon+\gamma h} \frac{1}{|\Gamma|} \int_{\Gamma} \boldsymbol{u} \cdot \boldsymbol{n} d \sigma \int_{\Gamma} \boldsymbol{v} \cdot \boldsymbol{n} d \sigma+ \\
& -\frac{1}{|\Gamma|} \int_{\Gamma} \mu \partial_{n} u_{n} d \sigma \int_{\Gamma} \boldsymbol{v} \cdot \boldsymbol{n} d \sigma-e^{-\varepsilon} \frac{1}{|\Gamma|} \int_{\Gamma} \mu \partial_{n} v_{n} d \sigma \int_{\Gamma} \boldsymbol{u} \cdot \boldsymbol{n} d \sigma \\
F^{P}(\boldsymbol{v})= & \int_{\Gamma} \boldsymbol{f} \cdot \boldsymbol{v} d \sigma+\frac{1}{\varepsilon+\gamma h} \frac{1}{|\Gamma|} Q \int_{\Gamma} \boldsymbol{v} \cdot \boldsymbol{n} d \sigma+ \\
& -e^{-\varepsilon} \frac{1}{|\Gamma|} Q \int_{\Gamma} \mu \partial_{n} v_{n} d \sigma-\frac{\varepsilon}{\varepsilon+\gamma h} P \int_{\Gamma} \boldsymbol{v} \cdot \boldsymbol{n} d \sigma .
\end{aligned}
$$

We have the following

Theorem 5. If Hypothesis 1 is satisfied, Problem 1, with $a^{P}(\cdot, \cdot)$ and $F^{P}(\cdot)$ instead of $a(\cdot, \cdot)$ and $F(\cdot)$, is consistent with respect to problem $(2)_{1,2,4^{-}}(20)$.

Proof. The proof follows the same guidelines of those of Theorem 1.

We highlight that no well-posedness results are available for system (6) with bilinear form $a^{P}(\cdot, \cdot)$ and functional $F^{P}(\cdot)$.

In the case $\varepsilon=0$ we obtain again the flow rate problem (12). If $\varepsilon \rightarrow \infty$, we obtain the following weak problem

$$
\left\{\begin{array}{c}
\mu \int_{\Omega} \nabla \boldsymbol{u}_{h}: \nabla \boldsymbol{v}_{h} d \boldsymbol{x}-\frac{1}{|\Gamma|} \int_{\Gamma} \mu \partial_{n} u_{h, n} d \sigma \int_{\Gamma} \boldsymbol{v}_{h} \cdot \boldsymbol{n} d \sigma-\int_{\Omega} p_{h} \nabla \cdot \boldsymbol{v}_{h} d \boldsymbol{x}= \\
=\int_{\Gamma} \boldsymbol{f} \cdot \boldsymbol{v}_{h} d \sigma-P \int_{\Gamma} \boldsymbol{v}_{h} \cdot \boldsymbol{n} d \sigma, \quad \forall \boldsymbol{v}_{h} \in\left[V_{h}\right]^{d}, \\
-\int_{\Omega} q_{h} \nabla \cdot \boldsymbol{u}_{h} d \boldsymbol{x}-\gamma h \frac{1}{|\Gamma|} \int_{\Gamma} p d \sigma \int_{\Gamma} q d \sigma=-\gamma h P \int_{\Gamma} q d \sigma, \quad \forall q_{h} \in Q_{h} .
\end{array}\right.
$$

Integrating by parts the first equation of (22), we obtain the following boundary condition

$$
\frac{1}{|\Gamma|} \int_{\Gamma}\left(\mu \nabla \boldsymbol{u}_{h} \boldsymbol{n}-p_{h} \boldsymbol{n}\right) d \sigma=\left(\frac{1}{|\Gamma|} \int_{\Gamma} \mu \partial_{n} u_{h, n} d \sigma-P\right) \boldsymbol{n} \quad \text { on } \Gamma,
$$

which, under Hypothesis 1 , is consistent with the mean pressure condition $(1)_{2}$. We observe that condition (23) arising from formulation (22) is different from the one obtained from the weak formulation proposed in [15], that is

$$
\frac{1}{|\Gamma|} \int_{\Gamma}\left(\mu \nabla \boldsymbol{u}_{h} \boldsymbol{n}-p_{h} \boldsymbol{n}\right) d \sigma=-P \boldsymbol{n} \quad \text { on } \Gamma
$$

which is not consistent with $(1)_{2}$ in the case of general geometries. 
We remark that, unlike system (6), the mean pressure problem (22) is not symmetric. Indeed, the $i j-t h$ entry of the velocity matrix is $\mu \int_{\Omega} \nabla \boldsymbol{\psi}_{j}: \nabla \boldsymbol{\psi}_{i} d \boldsymbol{x}-$ $\frac{1}{|\Gamma|} \int_{\Gamma} \mu \partial_{n} \psi_{j, n} d \sigma \int_{\Gamma} \boldsymbol{\psi}_{i} \cdot \boldsymbol{n} d \sigma$, where $\boldsymbol{\psi}_{j}$ 's are the velocity basis functions. More in general, formulation with $a^{P}(\cdot, \cdot)$ and $F^{P}(\cdot)$ and with $\varepsilon>0$ is not symmetric. This is strictly related to the fact that the mean pressure condition does not give any information about the viscous stress, but only about the pressure. In other words, this condition is "more than defective" since it does not prescribe an average information on the whole normal stress, but only on the pressure.

\section{Augmented formulation and algebraic settings for the flow rate problem}

In this section we address the flow rate problem, obtained from (1) by setting $\varepsilon=0$. In view of the numerical comparison presented in Section 6.1, we present here the algebraic counterpart of the Augmented formulation strategy introduced in [10] for the prescription of the flow rate for the steady Stokes problem and extended in [27] for the non-linear unsteady case. The Augmented formulation is obtained by introducing a Lagrange multiplier for each flow rate condition in the weak formulation. For the sake of simplicity, we consider a single flow rate and the steady Stokes problem. Then, the algebraic counterpart of the Augmented formulation reads

$$
\left[\begin{array}{ccc}
K & B^{T} & \boldsymbol{\Phi}^{T} \\
B & 0 & 0 \\
\boldsymbol{\Phi} & 0 & 0
\end{array}\right]\left[\begin{array}{l}
\boldsymbol{U} \\
\boldsymbol{P} \\
\Lambda
\end{array}\right]=\left[\begin{array}{c}
\boldsymbol{F} \\
\mathbf{0} \\
Q
\end{array}\right]
$$

where

$$
\begin{array}{ll}
K_{i j}=\mu \int_{\Gamma} \nabla \boldsymbol{\psi}_{j}: \nabla \boldsymbol{\psi}_{i} d \sigma, & B_{k l}=\int_{\Gamma} \varphi_{k} \nabla \cdot \boldsymbol{\psi}_{l} d \sigma, \\
F_{i}=\int_{\Gamma} \boldsymbol{f} \cdot \boldsymbol{\psi}_{i} d \sigma, & \phi_{j}=\int_{\Gamma} \boldsymbol{\psi}_{j} \cdot \boldsymbol{n} d \sigma .
\end{array}
$$

In (24), $\Lambda$ is the (unknown) Lagrange multiplier and $\varphi_{j}^{\prime} s$ are the pressure basis functions. We write system (24) in the following equivalent form (see [10]):

$$
\left[\begin{array}{cc}
A^{\Lambda} & \left(B^{\Lambda}\right)^{T} \\
B^{\Lambda} & 0
\end{array}\right]\left[\begin{array}{l}
\boldsymbol{U}^{\Lambda} \\
\boldsymbol{P}^{\Lambda}
\end{array}\right]=\left[\begin{array}{c}
\boldsymbol{F}^{\Lambda} \\
\mathbf{0}
\end{array}\right]
$$

where $A^{\Lambda}=\left[\begin{array}{cc}K & \boldsymbol{\Phi}^{T} \\ \boldsymbol{\Phi} & 0\end{array}\right], \boldsymbol{U}^{\Lambda}=\left[\begin{array}{c}\boldsymbol{U} \\ \Lambda\end{array}\right], \boldsymbol{P}^{\Lambda}=\boldsymbol{P}$ and $\boldsymbol{F}^{\Lambda}=\left[\begin{array}{c}\boldsymbol{F} \\ Q\end{array}\right]$, and where $B^{\Lambda}$ is obtained by adding a column of zeros to $B$. 
For the Nitsche's method (12), the algebraic problem reads

$$
\left[\begin{array}{cc}
A^{N} & \left(B^{N}\right)^{T} \\
B^{N} & 0
\end{array}\right]\left[\begin{array}{l}
\boldsymbol{U}^{N} \\
\boldsymbol{P}^{N}
\end{array}\right]=\left[\begin{array}{l}
\boldsymbol{F}^{N} \\
\boldsymbol{G}^{N}
\end{array}\right]
$$

where $A^{N}=K+S^{1}+S^{2}+\left(S^{2}\right)^{T}$, with

$$
\begin{array}{ll}
S_{i j}^{1}:=\frac{1}{\gamma h} \frac{1}{|\Gamma|} \int_{\Gamma} \boldsymbol{\psi}_{j} \cdot \boldsymbol{n} d \sigma \int_{\Gamma} \boldsymbol{\psi}_{i} \cdot \boldsymbol{n} d \sigma, & S_{i j}^{2}:=-\frac{1}{|\Gamma|} \int_{\Gamma} \mu \partial_{n} \psi_{i, n} d \sigma \int_{\Gamma} \boldsymbol{\psi}_{j} \cdot \boldsymbol{n} d \sigma \\
B_{k l}^{N}=b\left(\varphi_{k}, \boldsymbol{\psi}_{l}\right), & F_{i}^{N}=F\left(\boldsymbol{\psi}_{i}\right), \quad G_{k}^{N}=G\left(\varphi_{k}\right) .
\end{array}
$$

For the unsteady Oseen problem it is sufficient to add to matrix $K$ the term coming from time discretization and the non-linear term.

In view of the comparison in Section 6.1, we report here three different algorithms for the numerical solutions of the Augmented and of the Nitsche's methods.

- Monolithic solver;

- Pressure Schur complement equation

$$
B A^{-1} B^{T} \boldsymbol{P}=B A^{-1} \boldsymbol{F}-\boldsymbol{G},
$$

preconditioned with

$$
P^{C C}:=\left(\mu J^{-1}+\frac{1}{\Delta t} H^{-1}\right)^{-1}
$$

where $J$ is the pressure mass matrix and $H:=B L^{-1} B^{T}$. Denoting by $M$ the velocity mass matrix, $L$ is given by

$$
L=L^{\Lambda}=\left[\begin{array}{cc}
M & \boldsymbol{\Phi}^{T} \\
\mathbf{\Phi} & 0
\end{array}\right], \quad \text { for the Augmented strategy }
$$

or

$$
L=L^{N}=M+S^{1}+S^{2}+\left(S^{2}\right)^{T}, \quad \text { for Nitsche's strategy, }
$$

(Cahouet-Chabard preconditioner, CC, see [7]).

Once the pressure is known, the velocity is computed in the following way:

$$
A \boldsymbol{U}=\boldsymbol{F}-B^{T} \boldsymbol{P} .
$$

We point out that the construction of matrix $L$ for the Nitsche's method has been obtained by adding the penalty terms to the velocity mass matrix. For what concerns the matrix $J$, the use of the pressure mass matrix solely could be improved by taking into account the penalty terms. However, this approach is under investigation and in this paper we do not consider it; 
- Yosida method (see $[22,23])$ : Solve in sequence

$$
\begin{aligned}
& A \widetilde{\boldsymbol{U}}=\boldsymbol{F}, \\
& H \boldsymbol{P}=B \widetilde{\boldsymbol{U}}-\boldsymbol{G}, \\
& A \boldsymbol{U}=A \widetilde{\boldsymbol{U}}-B^{T} \boldsymbol{P} .
\end{aligned}
$$

The same remarks made for the matrix $L$ in the previous case hold as well.

\section{$6 \quad$ Numerical results}

The numerical simulations have been obtained by using a code written in Matlab. We have considered a backward Euler scheme for the time advance with a semiimplicit treatment of the convective term for the Navier-Stokes problem (see, e.g., [24]). We have discretized the fluid problems with $P 1 b u b b l e-P 1$ finite elements (see, e.g., [14]). The fluid viscosity and density have been set equal to 0.035 Poise and $1 \mathrm{~g} / \mathrm{cm}^{2}$. These values are taken from haemodynamic applications.

\subsection{Flow rate problem: A comparison with the Augmented formulation}

The accuracy of the solution obtained by prescribing a flow rate condition by means of the Nitsche's method (system (12)) has been pointed out in [29]. For this reason, we omit this part here and we focus on a real comparison with the Augmented formulation (for a discussion about the accuracy of the latter, we refer the reader to $[27])$.

\subsubsection{Criteria for the numerical comparison}

Given a strategy, namely the Nitsche's or the Augmented methods, there are several ways to implement it. These different ways are called in what follows algorithms. In view of a comparison between strategies, we introduce four comparison criteria.

- Dependence on parameters. The accuracy of a strategy could depend on the tuning of one or more parameters. The independence of any parameter is therefore a desiderable property of a strategy.

- Efficiency. Given two algorithms, an efficiency criterium is introduced by computing the CPU time to perform a given number of time steps. The same solver for the solution of the resulting linear systems and the same tolerances have to be used. A comparison between the two strategies is then performed by choosing the best algorithm (in term of CPU time) for each strategy. 
- Modularity. An algorithm is said to be modular if it can be implemented by resorting to available codes, for example commercial ones, used as blackboxes. A strategy is said to be modular if there exists at least one algorithm for its implementation which is modular.

- Generality. A strategy is said to be general if its formulation allows to treat other defective boundary conditions.

In what follows we present a comparison, based on the previous criteria, between the Nitsche and the Augmented methods for the prescription of the flow rate. In particular, we have chosen $\Omega=(0,3) \times(0,1), h=0.05$ and a time discretization parameter $\Delta t=0.01 \mathrm{~s}$. We have considered the unsteady Stokes and Navier-Stokes problems. We have imposed the flow rate

$$
Q=\cos (2 \pi t),
$$

at the inlet $\Gamma=\{x=0\} \times(0,1)$ of the rectangle, homogeneous Dirichlet conditions at the walls $\Gamma_{D}=(\{y=0\} \cup\{y=1\}) \times(0,3)$, and zero Neumann condition at the outlet $\Gamma_{\text {out }}=\{x=3\} \times(0,1)$. We observe that we have prescribed the pointwise conditions in the classical way, without resorting to the classical Nitsche's method.

\subsubsection{Dependence on parameters}

The imposition of the flow rate through the Nitsche's method depends on the tuning of the parameter $\gamma$ in the penalty term $S_{1}$ in (27). In this section we study the dependence of the accuracy of the prescribed flow rate on the choice of this parameter. We have considered the coniugate gradient (CG) method to solve the monolithic system (26).

In Table 1 we report the (percentage) $l^{1}$ error $\|\boldsymbol{E}\|_{1}$ for different values of $\gamma$, where $E_{j}:=\frac{\left|Q_{j}-Q_{j}^{N}\right|}{|Q|}$, and $Q_{j}$ and $Q_{j}^{N}$ are the prescribed and approximated by the Nitsche's method flow rates at time $t^{j}$. The final time is $T=0.40 \mathrm{~s}$. In

\begin{tabular}{ccccc}
$\gamma=3 \cdot 10$ & $\gamma=3 \cdot 10^{-1}$ & $\gamma=3 \cdot 10^{-3}$ & $\gamma=3 \cdot 10^{-5}$ \\
\hline & $48.43 \%$ & $36.16 \%$ & $1.26 \%$ & $0.01 \%$
\end{tabular}

Table 1: Mean relative error over 40 time steps.

Figure 2 we report the stream-wise velocity at the inlet at $t=0.20 \mathrm{~s}$ (left) and $t=0.30 \mathrm{~s}$ (right), for different values of the parameter $\gamma$. We have used the results obtained with the Augmented method as a reference solution (solid line in Figure 2).

The results reported in Figure 2 and Table 1 show the importance of tuning a suitable value for the parameter $\gamma$ in order to obtain an accurate solution with 

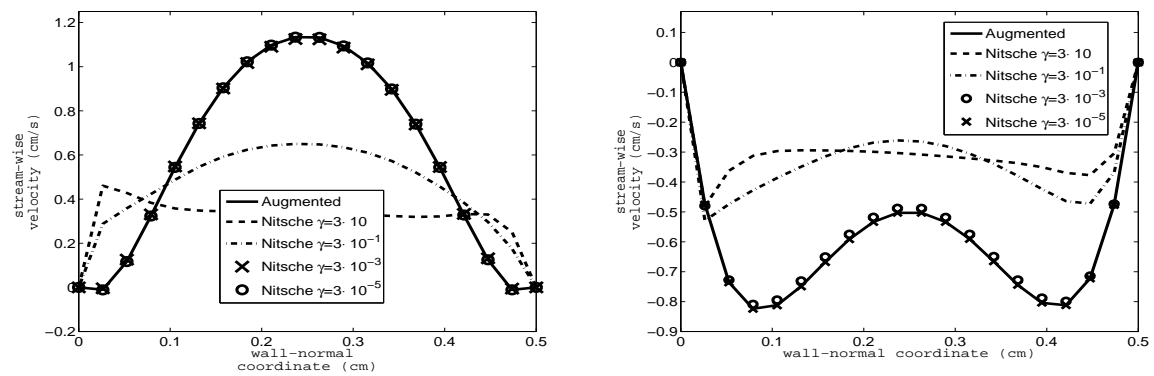

Figure 2: Stream-wise velocity at the inlet at time $t=0.20 \mathrm{~s}$ (left) and $t=0.30 \mathrm{~s}$ (right).

the Nitsche's method. On the contrary, the Augmented formulation is parameter independent.

For the numerical simulations presented in the continuation of Section 6.1, we have used $\gamma=3 \cdot 10^{-5}$.

\subsubsection{Efficiency}

We have applied the three different algorithms proposed in Section 5 to solve systems (25) and (26) for the unsteady Stokes problem. For the unsteady NavierStokes case we addressed only the performance of the monolithic and Yosida methods.

\begin{tabular}{ll|cc} 
& & Nitsche & Augmented \\
\hline \multirow{3}{*}{ Stokes } & Monolithic & $12 "(825.7)$ & $15 "(1152.6)$ \\
& CC & $216 "(7.4)$ & $18 "(7.6)$ \\
& Yosida & $94 ”(20.0+51.2)$ & $15 "(49.8+53.1)$ \\
\hline Navier- & Monolithic & $49 "(1101.7)$ & $77 " '(3088.1)$ \\
Stokes & Yosida & $128 ”(13.6+35.6)$ & $22 "(38.1+39.9)$
\end{tabular}

Table 2: CPU time in seconds to perform 20 time steps. In brackets, the mean number of subiterations per time step needed to reach convergence.

For the Stokes problem, we have used the CG method to solve the monolithic problems and the velocity subproblems in the CC and Yosida algorthms (relative tolerance equal to $10^{-8}$ ). We have explicitly built matrix $H$ in $\mathrm{CC}$ and Yosida methods, and we have solved the related systems with a multifrontal direct method (UMFPACK). The preconditioned CC pressure Schur complement equation has been solved with the Richardson method (relative tolerance equal 
to $\left.10^{-4}\right)$. For the Navier-Stokes case, we have used the Bicgstab method to solve the monolithic system and the velocity subproblems (again with relative tolerance equal to $10^{-8}$ ).

In Table 2 we report the CPU time in seconds to perform 20 time steps. In brackets we report the mean number of subiterations per time step to reach convergence (for $\mathrm{CC}$ we have reported only the number of Richardson's iterations, whilst for Yosida the number of iterations of the two velocity subproblems). From these results, we observe that the Nitsche's method is faster than the Augmented one when a monolithic approach is used, whilst it is slower for the other two proposed algorithms. In conclusion, we can state that for strategies which resort to a factorization, leading to the solution of subproblems in the velocity unknowns, the Augmented formulation seems to be more performing, whilst when one solves the problem monolithically, the Nitsche's method seems to be preferred.

\subsubsection{Modularity}

For what concerns the modularity, we observe that the Nitsche's method can not be modular, since all possible algorithms for its implementation need to build the penalization and boundary terms that appear in the variational formulation (12). Conversely, as pointed out in [10,27], the Augmented strategy is modular. Indeed, in [10] it has been proposed to write for the Stokes problem the Schur complement equation for the Lagrange multipliers. By applying the CG method to this equation, each residual computation leads to a "non Augmented" Stokes problem, which can be solved with an available (even commercial) code. In [27] it has been considered and analyzed the Navier-Stokes case, by introducing a GMRES algorithm for the Lagrange multipliers/Schur complement equation. In $[10,27]$ it has been observed that the number of residual computations, and then of Stokes solutions, needed to reach convergence in these algorithms is equal to the number of flow rate conditions plus one (the latter is requested for the computation of the initial residual). Therefore, even for a single flow rate, these strategies are non competitive from the efficiency point of view. For this reason, in [28] it has been introduced a new modular algorithm which reduces the computational costs to a single (Navier-)Stokes problem at each time step, introducing a boundary error at the artificial sections, which can be removed by slightly enlarging the computational domain.

Remark 3. In [29] a comparison concerning the efficiency of the Augmented and of the Nitsche's method for the Stokes problem has been presented by choosing for the first strategy the CG method applied to the Lagrange multiplier/Schur complement equation. This choice is not reasonable, since, as already pointed out, this algorithm has been introduced for modularity purposes and, for this reason, cannot be competitive from the efficiency point of view. In the author's opinion, the comparison should be done either by using the grouping of unknowns 
proposed in [10] (as done in this work, see system (25)), or by considering the efficient modular algorithm proposed in [28].

\subsubsection{Generality}

As shown in this work, the extended Nitsche's method is general, since it can be applied to a mean normal stress condition, to a mean pressure condition and to a linear combination of these with a flow rate condition. In particular, the proposed extended Nitsche's method (6) has the advantage that with a single implementation it is possibile to solve different defective problems, simply by tuning the parameter $\varepsilon$.

Conversely, as pointed out in $[10,13]$, the Augmented formulation can not be extended to the prescription of other defective conditions.

Remark 4. In [13] a different strategy for the solution of the flow rate problem, based on the control theory, has been introduced (control-based strategy). From the efficiency point of view, this strategy is not competitive with the Nitsche and the Augmented methods, since the monolithic problem has twice the degrees of freedom (for the presence of the adjoint problem). However, this strategy is modular for the Stokes case. In fact, by introducing an iterative method based on the steepest descend method, a sequence of classical Stokes solvers is introduced. Moreover, the control-based strategy is general, since mean pressure conditions can be treated as well.

\subsection{Validation of the complete formulation}

We present a validation of the proposed formulation (6) for the prescription, at the same time, of the flow rate and of the mean normal stress. In particular, we have considered the square domain $\Omega=(0,1) \times(0,1)$, the steady Stokes problem and rigid later walls $\Gamma_{D}=(\{y=0\} \cup\{y=1\}) \times(0,1)$. The Poiseuille's solution is given by

$$
\begin{gathered}
p(x, y)=1-x, \\
u(x, y)=\frac{y}{2 \mu}(1-y) .
\end{gathered}
$$

At the inlet $\Gamma=\{x=0\} \times(0,1)$, this solution features a flow rate and a mean normal stress given by

$$
\begin{aligned}
& Q=\frac{1}{12 \mu} \mathrm{cm}^{2} / \mathrm{s}, \\
& P=1 \text { dyne } / \mathrm{cm}^{2} .
\end{aligned}
$$

We observe that the normal stress coincides at the inlet with the pressure, so that the mean normal stress condition coincides with a mean pressure condition.

By setting $\gamma=10^{-3}, \Delta t=0.01$ and $h=0.05$, we have solved the steady Stokes problem with formulation (6), by prescribing the compatible data (28) at the inlet $\Gamma$ and a homogeneous Neumann condition at the outlet $\Gamma_{\text {out }}=\{x=$ 
$1\} \times(0,1)$. In Figure 3 we show the stream-wise velocity at the inlet and the mean pressure over the stream-wise direction, for different values of the parameter $\varepsilon$. These results show the excellent accuracy properties of the proposed method in comparison with the analytical solution and the robustness with respect to $\varepsilon$.
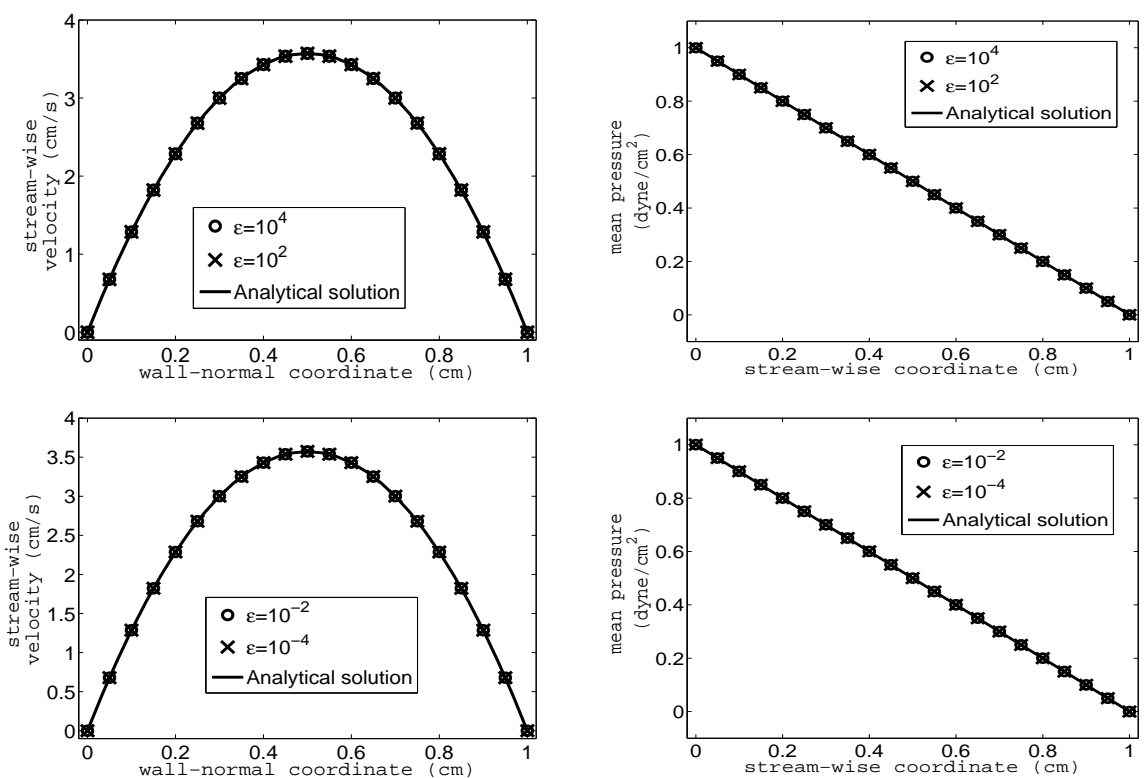

Figure 3: Extended Nitsche's method to prescribe the flow rate and the mean pressure at the same time. Stream-wise velocity at the inlet (left) and mean pressure (right) for different values of $\varepsilon$.

\subsection{The compliant case}

The two major difficulties in the solution of a fluid-structure interaction (FSI) problem are the satisfaction of the continuity conditions at the FS interface and the computation of the position of the fluid domain. For the first, one possible remedy consists in the solution of subsequent fluid and structure subproblems in an iterative way (partitioned procedures, see, e.g., [11, Chap.9]). In such a way, the continuity interface equations are satisfied thanks to the exchange of suitable interface conditions.

For what concernes the position of the fluid domain, one can consider an implicit treatment, by embedding the continuity equations' iterations in an external loop to update the FS interface position, or a semi-implicit one, by using, at each continuity equations' iteration, the fixed fluid domain obtained by extrapolation 
of previous time steps (see [8]).

In [12] it has been observed that the problem of prescribing the flow rate in the FSI case, can be easily treated by considering a partitioned procedure. Indeed, both for an implicit and for a semi-implicit treatment of the interface position, at each iteration of the partitioned procedure the fluid problem is solved in the $A L E$ configuration (see [16]) in a fixed domain. Therefore, it is sufficient to consider a partitioned procedure and to use one of the strategies introduced in the rigid case to prescribe the flow rate. Analogously, if the flow rate, the mean normal stress or both of them are prescribed, again we can consider a partitioned procedure and solve the fluid problem by using formulation (6).

In this section, we aim at validating the performance of formulation (6) in the FSI case. For the structure, we have considered the following equation of linear elasticity

$$
\rho_{s} \partial_{t t} \boldsymbol{\eta}-c \nabla \cdot\left(\nabla \boldsymbol{\eta}+(\nabla \boldsymbol{\eta})^{t}\right)-\lambda \nabla \cdot((\nabla \cdot \boldsymbol{\eta}) \boldsymbol{I})+\beta \boldsymbol{\eta}=\mathbf{0},
$$

where $\boldsymbol{I}$ is the identity operator, $c=E /(1+\nu), \lambda=\nu E /((1+\nu)(1-2 \nu))$ and $\beta=E /\left(1-\nu^{2}\right) R^{2}$, with $E$ the Young modulus, $\nu$ the Poisson ratio and $R$ the radius of the fluid domain. The reaction term stands for the transversal membrane effects. For its discretization, we have considered $P 1$ finite elements.

The fluid domain is a two-dimensional idealization of a realistic domain, namely the human carotid. Moreover, we have set $\Delta t=10^{-3} s, h=0.1, \rho_{s}=$ $1.1 \mathrm{~g} / \mathrm{cm}^{2}, c=1.15 \cdot 10^{6}$ dyne, $\lambda=1.7 \cdot 10^{6}$ dyne, $\beta=1.3 \cdot 10^{6}$ dyne $/ \mathrm{cm}^{2}$ and the thickness of the structure $H_{s}=0.1 \mathrm{~cm}$. At the generic outlet $\Gamma_{\text {out }}$, we have considered the following absorbing data in the defective boundary conditions $(1)_{1}$ and $(1)_{3}$

$$
\begin{aligned}
& P^{n+1}=\left(\left(\frac{\int_{\Gamma_{\text {out }}^{n}} \boldsymbol{u}^{n} \cdot \boldsymbol{n} d \sigma}{4 \delta A_{\text {out }}^{n}}+\left(A_{\text {out }}^{0}\right)^{1 / 4}\right)^{2}-\left(A_{\text {out }}^{0}\right)^{1 / 2}\right) \frac{\beta^{*}}{\sqrt{\pi}}, \\
& Q^{n+1}=4 \delta A_{\text {out }}^{n}\left(\sqrt{\frac{\sqrt{\pi} \frac{1}{\Gamma \mid} \int_{\Gamma} p d \sigma}{\beta}+\left(A_{\text {out }}^{0}\right)^{1 / 2}}-\left(A_{\text {out }}^{0}\right)^{1 / 4}\right),
\end{aligned}
$$

where $A_{\text {out }}$ is the area of the outlet and $\delta=\sqrt{\frac{\beta}{2 \rho_{f} \sqrt{\pi}}}$. Expressions (29) and (30) are absorbing boundary conditions, prescribed to avoid spurious reflections (see [21]). At the inlet, we have imposed the following flow-rate impulse

$$
F(t)= \begin{cases}50 \mathrm{~cm}^{3} / \mathrm{s} & t \leq 0.005 \mathrm{~s} \\ 0 & t>0.005 \mathrm{~s} .\end{cases}
$$

For the extended Nitsche's method, we have used $\gamma=10^{-10}$. In all the simulations, we have used the Robin-Neumann (RN) scheme as partitioned procedure 
(see [2]), a semi-implicit treatment of the FS interface position, and we have prescribed flow rates (31) at the inlet and (30) at the lower outlet.

In the first simulation we have prescribed the mean normal stress (29) at the upper outlet. The three defective data are imposed by means of the extended Nitsche's method (6). We have considered a monolithic solver for both the fluid and the structure subproblems. In Figures 4, the pressure in the deformed fluid domain and the exploded position of the structure are shown at 6 different instants.
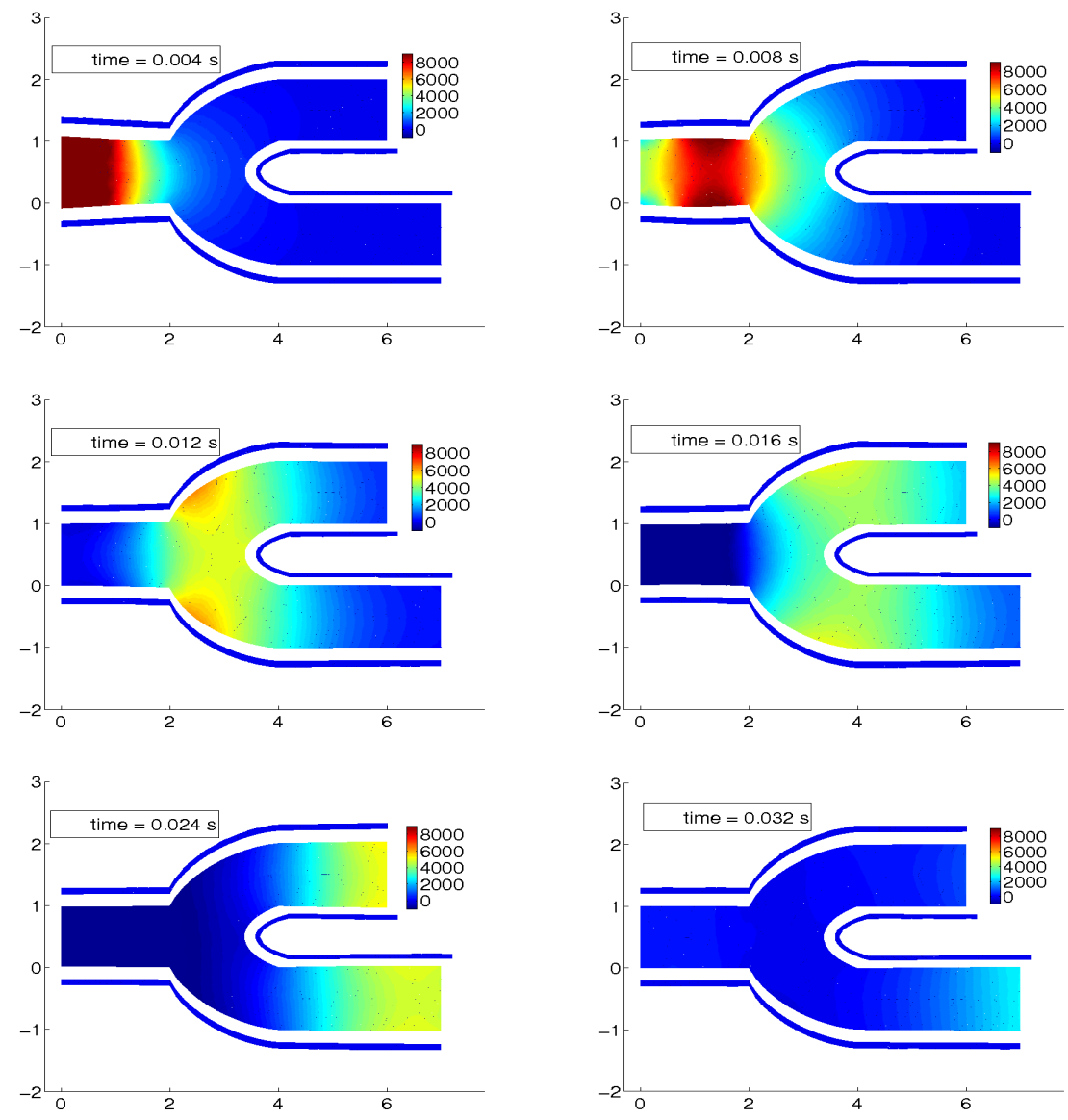

Figure 4: Extended Nitsche's method. Pressure in the deformed fluid domain and position of the structure.

Next, we have compared the performance of the extended Nitsche's method 
and of the Augmented formulation. We have prescribed mean normal stress (29) or flow rate (30) at the upper outlet. We point out that in the latter case we have prescribed the flow rate at each of the three artificial sections. This has been possibile since we have considered the RN scheme as partitioned procedure, which has been shown to guarantee the mass conservation law also when a Dirichlet or a flow rate condition is prescribed at the artificial sections (see [1]). For the Augmented strategy, the mean normal stress condition has been prescribed with a constant Neumann condition. Also fo the Augmented strategy we have considered a monolithic approach to solve the subproblems. In Table 3 we report the number of RN iterations and the CPU time needed to perform 32 time steps.

\begin{tabular}{l|cc} 
& extended Nitsche & Augmented \\
\hline Cond. (29) & $13.4-23^{\prime} 23^{\prime \prime}$ & $13.4-22^{\prime} 31^{\prime \prime}$ \\
Cond. (30) & $13.4-21^{\prime} 08^{\prime \prime}$ & $13.4-22^{\prime} 00^{\prime \prime}$
\end{tabular}

Table 3: Mean number of iterations per time step (left) and CPU time in minute $(')$ and seconds (") (right) to perform 32 time steps for the carotid simulation. We have considered two cases obtained by imposing condition (29) or (30) at the upper outlet.

From these results it follows that the extended Nitsche's method is an effective strategy to prescribe the flow rate and the mean normal stress also in the FSI case. Moreover, this strategy is competitive, in terms of efficiency, with the Augmented method, since the number of subiterations to reach convergence in the RN scheme does not seem to depend on the strategy used to solve the fluid subproblem. However, this example shows the great versatility of the proposed extended Nitsche's method, since, by using a single code and by properly tuning the parameter $\varepsilon$, it has been possible to switch easily from a defective problem to another.

\section{Conclusions}

In this paper we have proposed a unified treatment of defective boundary problems by means of the extended Nitsche's method. We have shown the wellposedness of this formulation and the convergence of the discrete solution towards the continuous one. We have focused on the differences between mean normal stress and mean pressure conditions, highlighting which are the corresponding consistent formulations. Moreover, we have performed a comparison between the proposed strategy and the Augmented one in the case of the flow rate problem. We have observed that the Nitsche's method performs better than the Augmented one in terms of generality, but not in terms of modularity and 
indipendence of parameters. From the efficiency point of view, with a monolithic approach the Nitsche's method seems to perform better, whilst with a factorization technique, the Augmented one seems to be preferred. We have then validate the proposed method when both the flow rate and the mean normal stress are prescribed at the same time, by a comparison with the Poiseuille solution. Finally, we have applied the proposed methodology to the fluid-structure interaction case, highlighting that the convergence of a partitioned procedure is independent of which strategy is used to prescribe the flow rate, and pointing out the versatility of the extended Nitsche's method in terms of implementation issues when one has to switch between different defective problems.

\section{Acknowledgments}

The author would like to thank P. Antonietti and A. Veneziani for fruitful discussions.

\section{References}

[1] S. Badia, F. Nobile, and C. Vergara. Robin-Robin preconditioned Krylov methods for fluid-structure interaction problems. Computer Methods in Applied Mechanics and Engineering, 198(33-36):2768-2784, 2009.

[2] S. Badia, F. Nobile, and Ch. Vergara. Fluid-structure partitioned procedures based on Robin transmission conditions. Journal of Computational Physics, 227:7027-7051, 2008.

[3] M. Benzi, H. G.H. Golub, and J. Liesen. Numerical solution of saddle point problems. Acta Numerica, pages 1-137, 2005.

[4] P.J. Blanco, R.A. Feijòo, and S.A. Urquiza. A unified variational approach for coupling 3d-1d models and its blood flow applications. Computer Methods in Applied Mechanics and Engineering, 196:4391-4410, 2007.

[5] F. Brezzi and M. Fortin. Mixed and hybrid finite element methods. Springer Verlag, 1991.

[6] F. Brezzi and J. Pitkäranta. On the stabilization of finite element approximation of the Stokes problem, in Efficient Solution of Elliptic Systems, W. Hackbush (Ed.), pages 11-19. Vieweg, Braunschweig, 1984.

[7] J. Cahouet and J.-P. Chabard. Some fast 3D finite element solvers for the generalized Stokes problem. International Journal for Numerical Methods in Fluids, 8:869-895, 1988. 
[8] M.A. Fernández, J.F. Gerbeau, and C. Grandmont. A projection semiimplicit scheme for the coupling of an elastic structure with an incompressible fluid. International Journal for Numerical Methods in Engineering, 69(4):794-821, 2007.

[9] L. Formaggia, J.-F. Gerbeau, F. Nobile, and A. Quarteroni. On the coupling of 3D an 1D Navier-Stokes equations for flow problems in compliant vessels. Computer Methods in Applied Mechanics and Engineering, 191(6-7):561$582,2001$.

[10] L. Formaggia, J.-F. Gerbeau, F. Nobile, and A. Quarteroni. Numerical treatment of defective boundary conditions for the Navier-Stokes equation. SIAM Journal on Numerical Analysis, 40(1):376-401, 2002.

[11] L. Formaggia, A. Quarteroni, and A. Veneziani (Eds.). Cardiovascular Mathematics - Modeling and simulation of the circulatory system. Springer, 2009.

[12] L. Formaggia, A. Veneziani, and C. Vergara. Flow rate boundary problems for an incompressible fluid in deformable domains: formulations and solution methods. Computer Methods in Applied Mechanics and Engineering, in press.

[13] L. Formaggia, A. Veneziani, and C. Vergara. A new approach to numerical solution of defective boundary value problems in incompressible fluid dynamics. SIAM Journal on Numerical Analysis, 46(6):2769-2794, 2008.

[14] V. Girault and P.A. Raviart. Finite element methods for Navier-Stokes equations. Springer-Verlag, 1986.

[15] J.G. Heywood, R. Rannacher, and S. Turek. Artificial boundaries and flux and pressure conditions for the incompressible Navier-Stokes equations. International Journal for Numerical Methods in Fluids, 22:325-352, 1996.

[16] T. J. R. Hughes, W. K. Liu, and T. K. Zimmermann. Lagrangian-Eulerian finite element formulation for incompressible viscous flows. Computer Methods in Applied Mechanics and Engineering, 29(3):329-349, 1981.

[17] T.J.R. Hughes, L.P. Franca, and M. Balestra. A new finite element formulation for computational fluid dynamics: V. Circumventing the BabuŝkaBrezzi condition: a stable Petrov-Galerkin formulation for the Stokes problem accommodating equal-order interpolations. Computer Methods in Applied Mechanics and Engineering, 59:85-99, 1986.

[18] M. Juntunen and R. Stenberg. Nitsche's method for general boundary conditions. Math. Comp., 78:1353-1374, 2009. 
[19] H.J. Kim, C.A. Figueroaa, T.J.R. Hughes, K.E. Jansen, and C.A. Taylor. Augmented lagrangian method for constraining the shape of velocity profiles at outlet boundaries for three-dimensional finite element simulations of blood flow. Computer Methods in Applied Mechanics and Engineering, 198:3551-3566, 2009.

[20] J.A. Nitsche. Uber ein variationsprinzip zur lozung von dirichlet-problemen bei verwendung von teilraumen, die keinen randbedingungen unterworfen sind. Abhandlungen aus dem Mathematischen Seminar der Universitat Hamburg, 36:9-15, 1970/71.

[21] F. Nobile and C. Vergara. An effective fluid-structure interaction formulation for vascular dynamics by generalized Robin conditions. SIAM Journal on Scientific Computing, 30(2):731-763, 2008.

[22] A. Quarteroni, F. Saleri, and A. Veneziani. Analysis of the Yosida method for the incompressible Navier-Stokes equations. Journal de Mathématiques Pures et Appliquées, 78:473-503, 1999.

[23] A. Quarteroni, F. Saleri, and A. Veneziani. Factorization methods for the numerical approximation of Navier-Stokes equations. Computer Methods in Applied Mechanics and Engineering, 188:505-526, 2000.

[24] A. Quarteroni and A. Valli. Numerical approximation of partial differential equations. Springer, 1994.

[25] V. Thomée. Galerkin finite element method for parabolic problems. Springer, Springer series in computational matehmatics, vol. 25, 2001.

[26] A. Veneziani. Mathematical and Numerical Modeling of Blood Flow Problems. PhD thesis, University of Milan, 1998.

[27] A. Veneziani and C. Vergara. Flow rate defective boundary conditions in haemodinamics simulations. International Journal for Numerical Methods in Fluids, 47:803-816, 2005.

[28] A. Veneziani and C. Vergara. An approximate method for solving incompressible Navier-Stokes problems with flow rate conditions. Computer Methods in Applied Mechanics and Engineering, 196(9-12):1685-1700, 2007.

[29] P. Zunino. Numerical approximation of incompressible flows with net flux defective boundary conditions by means of penalty technique. Computer Methods in Applied Mechanics and Engineering, 198(37-40):30263038, 2009. 